\title{
Robust Adaptive Control System Research
}

\author{
Gangyuan Mao ${ }^{1, a}$, Yingxian Yang ${ }^{2, b}$ \\ ${ }^{1,2}$ Huaiyin Institute of Technology, Electronic Information Engineering, Jiangsu Huai'an, 223003, \\ China
}

aemail: 13952381103@139.com, bemail: 870265905@qq.com

\begin{abstract}
Keywords: Adaptive Control; Robustness Duality; Generalized Predictive Control
\end{abstract}
\begin{abstract}
Because based on linear model, order and delay the known statistical rule, noise and other known some strict mathematical constraints (referred to as the "ideal") and design of adaptive control system under the complex outside disturbance of unmodeled dynamic characteristics and is often a lack of robustness, therefore since the $80 \mathrm{~s}$ study of robust adaptive control has received the widespread attention. This paper analyzes the traditional adaptive control system for lack of robustness and adaptive control system are reviewed in this paper the methods and research results of robustness, including algorithm is modified and the robust optimization and nonlinear adaptive control system design.
\end{abstract}

\section{Introduction}

Until the early 80s, the study of adaptive control system on the processing of the charged process mathematical model mostly has the following characteristics (referred to as the "ideal") :

(1) the dynamic characteristic of the thought process can be completely described by the linear deterministic or stochastic mathematical model, linear;

(2) assumes that the mathematical model of some key structural parameters (such as polynomial order, process, delay, etc.) when designing adaptive controller has been known;

(3) in the case of a stochastic model, tend to assume that ambient noise obey certain exactly known probability and statistics law, such as gaussian white noise;

(4) in order to establish the corresponding algorithm convergence and global stability of the control system, applying some restrictive conditions tend to process model, continuous incentive conditions, such as signal noise model of strictly positive real condition, input and output polynomial terms and condition of minimum phase polynomial co-prime, etc.

However, all sorts of practical industrial processes are running in a changing environment, at the same time, the people's understanding of the dynamic characteristics of a complex process of also is very limited and not comprehensive. Therefore, try to use the precise mathematical model to describe the dynamic characteristics of the process of completely is not realistic, it is impossible, the established mathematical model it describes the process of dynamic characteristics of a certain approximation, its approximation degree reflects the process model of the designer of the amount of knowledge and modeling techniques, rely solely on this mathematical model to establish the closed-loop adaptive control system is often difficult to achieve the desired control effect in the practical application.

1982, Rohrs and collaborators on a few adaptive control system performance were analyzed, and pointed out that when the charged process exists unmodeled dynamic characteristics, the adaptive control system will lose its stability properties (referred to as the "lack of robustness"), which caused the field the extensive study of robust adaptive control system. The significance of the study is that, in accordance with the requirements of robustness design of adaptive control system than just according to the convergence and stability index of the designed system has a wider application range and better control effect.

Due to lack of robustness of adaptive control system for many reasons, generally can be classified into three categories, one is charged process with unmodeled dynamic properties (especially when linear model), this is the main reason for the most part; The second is statistical 
properties of process noise or disturbance is far more complicated than it envisioned is; Three is the conditions stipulated in the "ideal" be broken. For example, as a result of the closed loop signal circuit characteristic, if the reference signal is too "single", as a control signal can't cover the dynamic characteristics of effective frequency band, continued incentive conditions can't meet, and this is often happen. Such as, many systems in addition to the hypothesis environmental noise is a random process with rational spectral density, also assume that is driven by the gaussian white noise process of linear regression, and then noise model satisfy the strictly positive real condition, this is unpredictable random noise characteristics of real industrial process is often difficult to achieve. So, for the convergence and stability of the results of the restrictions imposed by the process conditions relative to the actual situation of the process is too harsh. Certainly, because of the assumptions and conditions don't meet, designed based on the "ideal" adaptive control system of the instability is inevitable.

From dynamic behavior of the process of inspection, the most practical industrial process with nonlinear characteristics. Therefore, unmodeled dynamic characteristics must contain nonlinear ingredients, at this point, based on the linear model of closed-loop adaptive control system is designed a suboptimal solution, it is difficult to maintain stability and optimality of the entire operating range.

\section{Research Status}

Study of adaptive control system robustness roughly divided into three stages. The first phase of the researchers put forward a lot of counter example, points out the existing adaptive control system stability and control performance for modeling the dynamic characteristics and at the end of the observation noise and other highly sensitive; Second stage for lack of robustness of adaptive control scheme, especially slow adaptive scheme, the researchers on the mechanism of instability of detailed and extensive research, got some understanding. A large number of facts show that the existing adaptive control scheme for robustness analysis and robust design is the important way to solve the problem of instability; Research of the third stage is based on the previous research results, the adaptive robust control system design again, for some adaptive algorithms including the modified or new scheme is designed according to the robustness principle, in order to eliminate or partly eliminate the model does not accord with the process of mismatch (model) and does not meet the assumptions and cause instability of the closed-loop adaptive control system or performance degradation.

System ferreting about nonlinear system and that the study of adaptive control is adaptive control throughout the development process, and many theoretical achievements have been made. However, because of difficult to establish mathematical model and calculation reasons, can Enough to truly implement the established system is mostly based on linear model. In recent years, however, caused by unmodeled dynamic characteristics of the robustness problem of force researchers to reconsider the basic fact nonlinear process.

In the study of the robustness of control system, the h-infinity method made a great success. The h-infinity optimization theory and method is introduced in the field of adaptive control has become a unique, the strong vitality of the branch, and caused the researchers greatly Interest. In addition, originated in the control of the engineering application of the generalized predictive control (GPC) is also improved the traditional adaptive system optimization and control strategies, has strong robustness, and even into the application of industrial control behind P ID of one of the control algorithm.

\section{Research Methods and Results}

Improve robustness of adaptive control system way many, there is amended as characteristic of the robust design method, has been put forward to optimize index of the robust optimization method, a direct object by nonlinear model to a nonlinear method, have to expert system and neural network as the basic framework of intelligent method and so on. 


\section{The Robust Design Again}

Robust redesign of the basic idea is by limiting the adaptive loop gain in order to prevent the instability and reduce the phenomena of integral action to curb drift of process parameters, and has the following several kinds of method has been widely used in the literature: the use of dead zone or relative dead-zone method to limit the influence of noise on system disturbance in the dead zone; Using the normalized parameter estimation to make circuit signal remain in the sense of normalized signal boundedness; R2 correction algorithm in adaptive control law is to add a correction term, to eliminate the adaptive law of pure integral action, overcome the low frequency or high frequency unmodeled characteristics may cause the instability of; By using the projection algorithm parameter estimation is mapped to a designated area, in order to prevent infinite drift parameter estimation; Weakened by using average method to the requirement of strictly positive real condition; Using the method of attenuation incentive not a sufficient incentive to make up the circuit of signal.

\section{1) Dead Zone Modification Method}

Bounded disturbance and unmodeled dynamic characteristic parameter drift, will cause if the upper bound of the modeling error or disturbances is known, can be set in the adaptive control algorithm is a dead zone, nonlinear, only when the tracking error is beyond the dead zone, to generate the adaptive function, or interrupt the adaptive function.

\section{2) Signal Normalization Method}

Normalization of signal processing is the introduction of the characteristics of the normalized signals so that the adaptive circuits of all signals relative to the normalized mean bounded, overcome the amount due to the return signal parameter estimation algorithm of proliferation caused by the failure. This method first introduced by Praly, used to analyze the adaptive controller with robust stability of the time-varying parameter or modeling errors. Orte2ga the estimator of parameters such as the normalized processing, eliminates the problems because of the system are assumed to bounded, then use the fan stability theory and system of passive system stability theorem research. Design a when linear discrete system dynamic modeling error and has a foul one global stability of the adaptive controller, the method is adopted, the process of the augmented description, normalized L S algorithm and parameter adaptive stopping rule. Praly et using normalized and the combination of the projection method for a class of linear minimum variance self-tuning controller of random process robustness analysis, get the following conclusion: ideally to achieve optimal control and optimal tracking; In a closed loop system under a continuing lack of incentive conditions mean square stability; Have a band interference in the presence of modeling error and can still build the global robust stability of the mean square bounded. Radenkovic and $\mathrm{M}$ chel also adopt the same method of random adaptive control system were analyzed, and proved that when the presence of unmodeled dynamic characteristics and with outside interference, the resulting adaptive controller can make the closed-loop system to keep up L in the sense of global stability and tracking error minimum, at the same time don't meet is solid assumptions and constant encouragement have the characteristics of the stable condition.

\section{3) Parameter Estimation of the Projection}

To prevent caused by external disturbance and model error estimate parameters of drift, every step of the parameter estimation will contain parameter projection to a true parameters of bounded convex domain, this method is the result of geometric analysis of the parameters on discerniblile algorithm. Ydstie has carried on the limits to process model (modeled the boundedness of the inverse stability, disturbances, etc), and then use the method of projection gradient estimation and parameters for the research of adaptive control scheme for the robust stability and robust transient response analysis, points out its in L up sense is robust stability, and transient response and the tracking error is in the L 2 sense with the modeling error or out of the ceiling with the order. The normalization method, pointed out some of the dead zone method, Wen and Hill only using the parameter estimation of orthogonal projection makes the adaptive controller has strong robustness. Ossman and Keman with another interval projection method to get the similar results. A classic RLS algorithm is introduced in the nonlinear judgment of prediction error method (called M 2) used for Clarke2Gawthrop self-tuning controller is used to estimate parameters, so as to eliminate the 
interference to the negative impact of the closed-loop system and the global stability and asymptotic optimality.

\section{4) R2 Correction Method}

Some scholars put forward in the adaptive control law by using a weighted correction term (R2) to suppress the because of outside interference and modeling error caused by the impact on the closed loop system, ensure the adaptive circuits of all signals are bounded, and get a small tracking error. The results show that whether the signal constant encouragement, R2 error correction method can make the parameter vector index attenuation to a collection of smaller, but the method may introduce unstable balance, resulting in "burst" phenomenon. R2 correction algorithm has many variants and improvements, including one called e12 at 11:45 correction algorithm, its advantage lies in the state of equilibrium under continuous excitation signal has the exponential stability, and the response characteristics.

\section{5) Average Method}

Average method for parameter changes the state of much slower process (this feature is inherent in the adaptive problems, otherwise it will be difficult to process is described by using parametric characteristics), the circuit by its within a certain period of time averaged to reduce the order of the process, reasonably and weakening demand for strictly positive real condition to achieve inhibition caused by external disturbance and model error and parameter perturbation. This method in the analysis of the robustness of adaptive control has been widely applied.

\section{6) Attenuation Incentive Method}

Attenuation in the process of incentive method is to solve the actual signal insufficient incentive and artificially in the reference trajectory control signal or join a exponential convergence of small excitation signal, to achieve the purpose of various modal characteristics of the excitation process. This method brings the biggest advantage is that not only can make the system B IBO is stable, and the process parameters converge to the true value. Chen and Guo motivation techniques using attenuation proved that: if the unmodeled dynamic characteristics is a small constant $\mathrm{E}$ multiplied by a process depends on the regression vector quantity control, the control quantity increases with the increase of them can be infinite growth, the estimated error and the tracking error will be proportional to the upper limit of modeling error and external disturbance. Radenkovic first in-depth analyses the shortcoming of the traditional adaptive control system, the finite energy external motivation and calm by the parameter drift and the numerical calculation of zero except the instability caused by system, and the estimated error limits.

\section{The Robust Optimization Method}

Robust optimization method for traditional optimization theory is the major concept of the controller robustness is not strong, as a result of the shortcomings, to optimize proposition, overcome the negative influence of system uncertainties and modeling errors. This kind of method is the most representative in the dual adaptive control, h-infinity control and generalized predictive control.

\section{1) The Dual Adaptive Control}

Dual adaptive control strategy through the optimization performance index functional explicitly add item to reflect the system parameter uncertainties learning effect to put forward the corresponding optimization problem, to achieve the goals of both the control and learning system uncertainty, thus can greatly improve the robustness of the closed-loop system.

\section{2) Adaptive h-infinity Control}

Although h-infinity optimal controller design results continue to increase, but there are still many problems between the theory and application to be solved, the first is the amount of calculation problem. Because the h-infinity optimal controller is generally complicated structure, order, so under the condition of existing technology brought great difficulties to the implementation. In addition, the h-infinity optimal controller generally requires a precise mathematical model, the process of giving process parameters are unknown or partially unknown or time-varying with great difficulty, which is difficult to apply online on discerniblile ideas and methods. 


\section{3) The Generalized Predictive Control (GPC)}

In most researchers focus on how to use the robust redesign and h-infinity optimization index to improve the robustness of adaptive control system, the others are more concerned with the researchers of industrial process control application is trying to break the traditional way of constraints, in the face of the characteristics of the industrial process, looking for a variety of low requirements for model, comprehensive control of good quality and new algorithm of optimal control convenient, online calculation of generalized predictive control (GPC) is one of the most successful.

In predictive control, the optimization is not an offline, but online again and again, so that when the system is uncertainty, according to the prediction of the moment on the system uncertainty to develop a suitable for the optimization of this moment, to avoid the global optimization of fixed index brings to the system of decision-making errors, this also is the main cause of GPC robust. Though predictive control research starts relatively late, but have a lot of achievements and progress rapidly, even have been some software of control system company to its products, successfully application. Of course, with a variety of extensive research of the GPC algorithm about the stability, convergence and robustness of the theoretical analysis results are also beginning to set up.

\section{The Nonlinear Method}

Because there are many difficulties to deal with nonlinear system, so that often use global or local linearization method for analysis, synthesis and control of the system. The weak nonlinear system, the linear model to a certain extent, can be well close to the original system, but about the nature of the strongly nonlinear or nonlinear systems, especially when the large range of circuit signal changes and frequently, the approximation model is hard to control performance with satisfied precision. As a result, nonlinear system modeling, identifying, analyzing, comprehensive and adaptive control has become an important branch of control theory.

\section{Intelligent Methods}

Intelligent method breaks through the traditional concept and technology, using a computer the tool in such aspects as reasoning, learning, and computational advantages, will be the idea of intelligent control and combine the principle of adaptive opens up a new way. The method is basically divided into two kinds, one is based on expert system characteristics of stratified hierarchical system, the second is based on neural network model and optimization method. Due to the practical problems in the successful application of intelligent control, adaptive control system of intelligent methods it has received extensive attention.

\section{Summary}

Due to a lack of robustness, the traditional adaptive control system had a lot of difficulties in the practical application, and again by the robust design, the robust optimization principle and intelligent idea of robust adaptive control system is characterized by control engineers welcomed, and caught the attention of the theory researchers. As you might expect, from application field, the study will continue to proceed in depth.

\section{References}

[1] Rohrs C E, et al. The Robustness of Adaptive Control Algorithm s in the Presence of Unmodelled Dynamics.(2002)

[2] J. Astrom K the Adaptive Feedback Control. The Proc IEEE. Commun 25(1987)185-217.

[3] Ioannou argues P. A, Sun j. and found the and the Design of Robust Direct and Indirect The Adaptive Control Schemes. Int. J. Control, 1988, pp.775-813.

[4] Yuan Zhu zhijun Wei-min wang Chen enhancement Some progress of robust self-tuning controller control theory and application.(2007)

[5] Praly l. Robustness of an Indirect Adaptive Control -based on Po le Placement. The Design P roc IFAC Work shop on the Adaptive Control.(2008) 